\title{
Contribution of dedifferentiated proximal tubule cells to repair in acute kidney injury
}

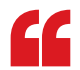

all injured

PTECs have an equivalent capacity to repair

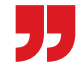

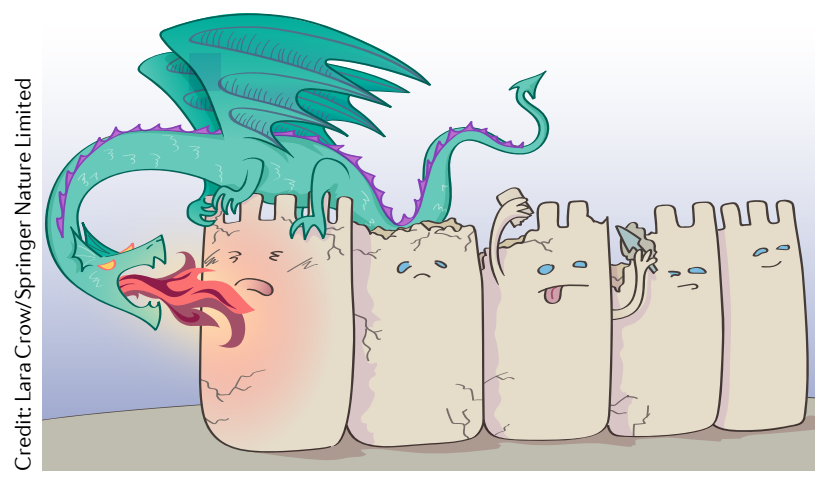

KIM1 is undetectable in healthy PTECs but is rapidly induced in response to injury, allowing the researchers to determine the fate of individual, injured Kim $1^{+}$PTECs.

"The ability to track the fate of injured cells, and quantitate the fraction that go onto successful repair versus those that fail to repair, is a completely new and highly valuable approach to better understand successful rather than failed repair in relation to the transition of acute injury to chronic kidney disease," explains Humphreys.

Lineage tracing of genetically labelled cells revealed clonal expansion of $\mathrm{Kiml}^{+}$cells up to 14 days after ischaemia-reperfusion injury (IRI). This expansion coincided with indices of renal recovery, indicative of a proliferative repair process. Further analyses showed that although the majority of labelled PTECs underwent complete repair, approximately $15 \%$ of labelled cells showed evidence of persistent injury, indicative of an incomplete repair process.

The researchers then performed transcriptional profiling of $\mathrm{Kim}^{+}$ PTECs to identify molecular processes involved in injury and repair. Analysis of differential gene expression patterns showed changes in the levels of 1,457 genes between day 2 and 7 following IRI, which subsequently returned towards baseline levels by day 14 . Gene ontology analysis showed upregulation of genes involved in the cell cycle, DNA repair, the immune response and cilium assembly at day 7 compared with day 2 . By contrast, genes that were upregulated by day 14 included those involved in cell transport and metabolic processes, indicating redifferentiation of these cells.

Foxm1, which is involved in cell cycle progression in high turnover organs, was one of the most highly upregulated transcription factors in injured PTECs. To assess its role, Chang-Panesso and colleagues used small interfering RNA to knockdown expression of FOXM1 in cultured human PTECs. Downregulation of FOXM1 resulted in impaired PTEC proliferation, suggesting this transcription factor is involved in cell cycle progression in PTECs. Inhibition of EGFR with the inhibitor erlotinib also suppressed expression of FOXM1 and its downstream targets in cultured human PTECs. Finally, administration of erlotinib following the induction of IRI in mice attenuated Foxm 1 expression and cell proliferation in a straindependent manner, indicating that EGFR regulates Foxm1 expression after injury in vivo.

Chang-Panesso says that their findings provide additional evidence that the surviving injured tubular epithelial cells contribute to repair and define a transcriptional signature for successful versus failed repair. "We now want to gain a deeper understanding of the transcriptional changes occurring in these injured PTECs in order to identify therapeutic strategies that will accelerate successful repair and prevent maladaptive repair leading to fibrosis and chronic kidney disease after an episode of acute kidney injury," she adds.

Susan J. Allison

ORIGINAL ARTICLE Chang-Panesso, $M$. et al. FOXM1 drives proximal tubule proliferation during repair from acute ischemic kidney injury. J. Clin. Invest. https://doi.org/10.1172/JCl125519 (2019) 